\title{
Análisis de parámetros en señales electrocardiográficas de pacientes con Chagas y de variables del dominio del tiempo de la HRV usando polígrafo de alta resolución
}

\author{
Analysis of parameters in electrocardiographic signals of patients with \\ Chagas and time domain variables of the HRV using high resolution \\ polygraph
}

\section{Análise de parâmetros em sinais eletrocardiográficos de pacientes com variáveis Chagas e de domínio do tempo da VFC utilizando polígrafo de alta resolução}

\author{
José R. Corredor-Matus ${ }^{1 *}$; Fernando Riveros-Sanabria ${ }^{2 *}$
}

\footnotetext{
1 MVZ, MSc, Facultad de Ciencias Agropecuarias y Recursos Naturales, Universidad de los Llanos, Colombia

2 Ing. Elec, MSc, cPhD, Facultad de Ciencias Básicas e Ingeniería, Universidad de los Llanos, Colombia

Grupo de Investigación MACRYPT, Universidad de los Llanos, Colombia

Email: jcorredor@unillanos.edu.co
}

Recibido: 08 de noviembre de $2017 \quad$ Aceptado: 17 de septiembre de 2019

\begin{abstract}
Resumen
Este trabajo presenta los resultados obtenidos de un estudio sobre el análisis de parámetros electrocardiográficos en registros de pacientes seropositivos a la enfermedad de Chagas del Departamento del Meta y medición de las variables en el dominio de tiempo de la variabilidad de la frecuencia cardiaca (HRV) y su comparación con registros de población de control. Se obtuvieron un total de 38 registros de 10 minutos en su gran mayoría (57,9\%) con un polígrafo de alta resolución entre ambas poblaciones, creándose una base de datos anotada de éstos registros. El presente estudio, permitió encontrar diferencias significativas de parámetros electrocardiográficos para el intervalo QTc entre los grupos, que podría ser consecuencia de alteración de la condución cardiaca que se traduce en un mayor incremento del QTc, así como de afectación de las variables del dominio de tiempo de la Variabilidad de la Frecuencia Cardiaca (HRV), como la SDRR y SEHR, que resultan en valores más bajos para los pacientes seropositivos a enfermedad de Chagas. Se concluye que variables de los registros ECG y de la HRV, presentan alteración en pacientes con enfermedad de Chagas, que podrían ser utilizados como alertas tempranas de la enfermedad no diagnosticada serológicamente.
\end{abstract}

Palabras clave: Enfermedad de Chagas, parámetros electrocardiográficos, Variabilidad de la frecuencia cardiaca (HRV), intervalo QTC.

Como Citar (Norma Vancouver):

Corredor-Matus JR, Riveros-Sanabria F. Análisis de parámetros en señales electrocardiográficas de pacientes con Chagas y de variables del dominio del tiempo de la HRV usando polígrafo de alta resolución. Orinoquia, 2019; 23(2):47-55. DOl:https://doi.org/10.22579/20112629.568 


\begin{abstract}
This paper presents the results obtained from a study on the analysis of electrocardiographic parameters in records of patients seropositive to Chagas disease of the Department of Meta and measurement of the variables in the time domain of heart rate variability (HRV) and its comparison with population control records. A total of 38 10-minute records were obtained in the vast majority $(57.9 \%)$ with a high resolution polygraph between both populations, creating an annotated database of these records. The present study allowed us to find significant differences in electrocardiographic parameters for the QTc interval between the groups, which could be a consequence of an alteration of the cardiac conduction that translates into a greater increase in the QTc, as well as the affectation of the time domain variables of Heart Rate Variability (HRV), such as the SDRR and SEHR, which result in lower values for HRV-positive patients with Chagas disease. It is concluded that variables from the ECG and HRV registries present an alteration in patients with Chagas disease, which could be used as early warnings of the serologically undiagnosed disease.
\end{abstract}

Key words: Chagas disease; electrocardiographic parameters; heart rate variability (HRV); QTc interval

\begin{abstract}
Resumo
Este artigo apresenta os resultados obtidos em um estudo sobre a análise de parâmetros eletrocardiográficos em prontuários de pacientes soropositivos para a doença de Chagas do Departamento de Meta e mensuração das variáveis no domínio do tempo da variabilidade da freqüência cardíaca (VFC) e sua comparação com os registros de controle populacional. Um total de 38 registros de 10 minutos foi obtido na grande maioria $(57,9 \%)$ com um polígrafo de alta resolução entre as duas populações, criando um banco de dados anotado desses registros. O presente estudo permitiu encontrar diferenças significativas nos parâmetros eletrocardiográficos para o intervalo QTc entre os grupos, o que pode ser consequência de uma alteração da condução cardíaca que se traduz em maior aumento no QTc, bem como no comprometimento das variáveis no domínio do tempo Variabilidade da Frequência Cardíaca (VFC), como o SDRR e o SEHR, que resultam em valores mais baixos para pacientes HIV positivos com doença de Chagas. Concluise que as variáveis dos registros de ECG e HRV apresentam alteração nos pacientes com doença de Chagas, que pode ser utilizada como alerta precoce da doença sorologicamente não diagnosticada.
\end{abstract}

Palavras chave: Doença de Chagas; Parâmetros do ECG; variabilidade da frequência cardíaca (HRV); intervalo QTc

\section{Introducción}

Actualmente, se estima que en América Latina existen alrededor de los infectados por la enfermedad de Chagas alcanzarían unos 6 a 7 millones de personas infectadas por la enfermedad de Chagas, de las cuales más del 30\% podría desarrollar síntomas de enfermedad crónica, en especial la cardiopatía, que provoca alrededor de 12.000 muertes por año. (PHAO/WHO, 2017). El estimativo de prevalencia de la infección por Trypanosoma cruzi, agente etiológico de esta enfermedad, en Colombia es de aproximadamente 436.000 habitantes, con un total de 5.250 casos nuevos anuales y una población expuesta en la zona endémica de 4.792.000, (Instituto Nacional de Salud y Organización Panamericana de la Salud, INS/OPS, 2017). Según los datos históricos del INS, siendo Casanare y Meta, dos de los departamentos más comprometidos por esta infección (Guhl, 1998).

En lo que tiene que ver con las manifestaciones clínicas de la enfermedad de Chagas, esta se caracteriza por presentar una fase aguda y una fase crónica, los cuales difieren en los signos clínicos y tasas de mortalidad. En la fase crónica la enfermedad se caracteriza por una reducida parasitemia y lesiones típicas en corazón y tubo digestivo, siendo la cardiopatía chagá- sica, el signo clínico más frecuente. (Andradre et al., 1998). Un estudio llevado a cabo en 120 pacientes diagnosticados con enfermedad chagásica, que ingresaron a por la clínica fundación Shaio de Bogotá, Colombia, reportó que las principales manifestaciones electrocardiográficas fueron: bloqueo completo de rama derecha $(40,0 \%)$, bloqueo aurículo-ventricular de segundo y tercer grado $(29,2 \%)$, disfunción del nodo sinusal $(28,3 \%)$, taquicardia ventricular $(23,0 \%)$, fibrilación auricular $(19,0 \%)$, hemibloqueo anterior izquierdo $(17,2 \%)$, flutter auricular $(3,3 \%)$ y bloqueo de rama izquierda (3,3\%) (Mora et al., 1998). Estos hallazgos son similares a los reportados por (Lázzari, 1994; Luquetti, 1997 Y Rosas et al., 2002). Del mismo modo, también se ha observado que la enfermedad genera disfunciones de la actividad autónoma, relacionadas con el incremento del tono simpático y denervación cardiovagal, que pueden cuantificarse a través de métodos de medición de la variabilidad de la frecuencia cardiaca (HRV) (Tundo et al., 2005).

La HRV es un parámetro definido como el intervalo de tiempo de un latido en un análisis de periodo de tiempo determinado (Sánchez et al., 2013; Rasmussen et al., 2014). Su determinación está basada en la medición de ondas $\mathrm{R}$ consecutivas y el cálculo en milisegundos (ms) del tiempo entre ellas. (Bogucki y 
Nosczczyk-Nowak, 2015; Rodas et al., 2008; Buzzano et al., 2012; Khor et al., 2014 ). La HRV se evalúa a través de parámetros de dominio temporal, de frecuencia y medidas no lineales (Rodas et al., 2008; Rasmussen et al., 2014). Los de tiempo son resultantes de la medición de los intervalos RR, que se ven afectados por la acción del sistema nervioso autónomo. Los otros parámetros que caracterizan a la señal electrocardiográfica (ECG) se deben a los reflejos de la actividad eléctrica del corazón y son: el intervalo de tiempo del inicio de la onda $\mathrm{P}$ hasta el final de la onda $\mathrm{Q}$ o inicio de la onda $\mathrm{R}$; intervalo PR que incluye la despolarización auricular y el retraso fisiológico del estímulo a su paso por el nodo auriculoventricular, el inicio de la onda Q hasta el final de la onda $S$ (intervalo del complejo QRS), intervalo RR, intervalo QT, e intervalo QT corregido (QTc), amplitud de la onda P, amplitud de la onda R, frecuencia cardiaca. Dado que la cardiopatía chagásica genera disfunciones de la actividad del sistema nervioso autónomo y esta disfunción puede valorarse con el estudio de la HRV, el análisis de esta variable en el dominio temporal, podría ser utilizada para identificar pacientes con cardiopatía, que no hayan sido diagnosticados con enfermedad de chagas y para quienes la valoración de la HRV podría ser utilizada como alerta temprana, para posteriormente comprobar la enfermedad a través de los exámenes serológicos correspondientes.

Por lo descrito anteriormente, se planteó como objetivo realizar un análisis de parámetros electrocardiográficos y del dominio de tiempo de la HRV de pacientes diagnosticados con enfermedad de Chagas y compararlos con población control, identificando resultados de estos parámetros que permitan ser utilizados para identificar pacientes no diagnosticados como chagásicos.

\section{Metodología}

El presente estudio se realizó según lo establecido en la resolución 8430 de 1993, artículos 11 y 15 del Minsalud, y la ley 842 de 2003 del código de Ética Profesional para el ejercicio de la Ingeniería Biomédica.

La toma de registros se ejecutó en el laboratorio de fisiología de la Universidad de los Llanos $\left(4^{\circ} 4^{\prime} 30^{\prime \prime} \mathrm{N}\right.$, $\left.73^{\circ} 35^{\prime} 7^{\prime \prime} \mathrm{W}\right)$, localizado a 4 kilómetros de la ciudad de Villavicencio, en la vereda Barcelona, cuyas características climatológicas son: temperatura media anual de $25^{\circ} \mathrm{C}$, precipitación anual media de $4050 \mathrm{~mm}$, humedad relativa promedio de $75 \%$ y altura sobre el nivel del mar de $420 \mathrm{~m}$.
La población experimental, fueron el total de pacientes diagnosticados por EC, registrados en la base de datos de la Secretaría Departamental de Salud del Meta. De ésta base de datos se seleccionaron aleatoriamente 50 pacientes, los cuales fueron contactados personalmente, con el fin de explicarles los objetivos del proyecto y poder recolectar los datos electrocardiográficos. Los pacientes contactados residían en diferentes municipios del departamento del Meta, razón por la cual solo se incluyeron aquellos pacientes que habitaban en el área urbana y rural de la ciudad de Villavicencio, 19 en total. Los individuos control fueron 19, quienes manifestaron no padecer ninguna enfermedad en el momento de realizar los registros electrocardiográficos. Los pacientes seropositivos tienen mayor edad, dado que la fase crónica de la enfermedad, cuando afecta el corazón, se desarrolla y diagnostica años después de la infección del paciente, cuando este asiste a la consulta por cardiopatía, no por enfermedad de Chagas.

\section{Toma y procesamieno de registros eletrocardiográficos}

Los pacientes incluidos en el estudio fueron trasladados de manera individual a las instalaciones del laboratorio de Fisiología de la Escuela de Ciencias Animales de la Universidad de los Llanos. Se le explicó a cada uno de ellos el objetivo del estudio y el procedimiento a realizar para la toma de los registros electrocardiográficos. Una vez obtenida su aprobación, leído y firmado el consentimiento informado, en el cuál cada individuo aceptó su participación en este estudio, el paciente fué ubicado en un cubículo independiente, aislado dentro de las instalaciones del laboratorio, a fin de tener la mayor comodidad y privacidad. La ubicación de los electrodos, se realizó siguiendo la guía para la obtención de la derivada bipolar DII, utilizando electrodos desechables, los cuales fueron descartados luego de su primer uso. Todas las pruebas de registro fueron realizadas por una profesional de enfermeria

Para la obtención del registro se utilizó un polígrafo ADinstruments de 8 canales referencia Power Lab $8 / 30$, con una resolución de 62,5 nanovoltios, escala de $+/-2 \mathrm{mV}$, ruido de $2,2 \mu \mathrm{V}$, filtro pasa-bajos de 0 $-50 \mathrm{~Hz}$, y una velocidad de muestreo de 2000 muestras/segundo, dotado de un acondicionador para ECG acoplado a un bioamplificador con puerto DIN, con entrada de tres electrodos. La visualización del registro se efectuó a través de la conexión del polígrafo con un computador portátil Asus, que además contiene el software Lab Chart de ADInstruments para el 
procesamiento de la información. Es debido a la alta resolución que solo se utilizó la derivada D II, con un rango de tiempo de muestreo entre 3 y 10 minutos de duración.

En el presente estudio solo se tuvieron en cuenta los valores de Frecuencia Cardiaca (HR), intervalo RR, intervalo QRS, intervalo QT e intervalo QTc, los cuales se utilizaron para el análisis correspondiente. Estos reportes se almacenaron en un procesador y se exportaron al programa Excel. Para la obtención de los parámetros de HRV, la información almacenada en Excel se exportó al software KUBIOS versión 2.1 de University of Eastern Finland, Kuopio (Finlandia), el cual procesa los datos y reporta los parámetros de la HRV: SDRR y SDHR. Tanto los parámetros electrocardiográficos reportados por Labchart Pro como los de HRV reportados por Kubios se organizaron en un archivo Excel para posteriormente someterlos al tratamiento estadístico.

\section{Procesamiento estadístico}

El procesamiento estadístico se realizó en el programa SPSS con el cual se obtuvo inicialmente la estadística descriptiva tanto de los parámetros electrocardiográficos como de HRV a los dos grupos incluidos en el estudio (Seropositivos a Chagas y controles). Posteriormente se efectuó un análisis de varianza de una vía a los dos parámetros, para establecer diferencias significativas entre ellos. Para determinar diferencias significativas entre grupos se corrió una prueba $\mathrm{T}$ de muestras independientes. En las tablas de resultados se incluyen promedios \pm SD. Se consideraron diferencias significativas a valores de $\mathrm{P} \leq 0.05$

\section{Resultados}

Un total de 38 personas fueron incluidas en el estudio, de los cuales el 50\% correspondió a seropositivas a la infección por $T$. cruzi, y el resto a personas pertenecientes al grupo control. Dentro del grupo de persona seropositivas a $T$. cruzi, se analizaron un total de 13 hombres y 6 mujeres, con una edad media de 52 años en el conjunto de la población (Tabla 1), mientras que para el grupo control se analizaron 15 hombres y 4 mujeres con una edad media del total de la población de 32 años (Tabla 2)

Las tablas 3 y 4 muestran los promedios de las variables electrocardiográficas medidas en cada uno de los grupos.

Como se observa en las tablas 3 y 4 , el promedio de la frecuencia cardiaca (HR) entre grupos no presentó diferencia estadísticamente significativa, sin embargo,
Tabla 1. Individuos seropositivos a Chagas

\begin{tabular}{|l|c|c|c|}
\hline $\begin{array}{c}\text { Código de } \\
\text { paciente }\end{array}$ & Sexo & $\begin{array}{c}\text { Edad } \\
\text { (años) }\end{array}$ & $\begin{array}{c}\text { Tiempo Registro } \\
\text { (min) }\end{array}$ \\
\hline H001C22 & M & 22 & 10 \\
\hline M002C49 & F & 49 & 10 \\
\hline M003C58 & F & 58 & 10 \\
\hline H004C53 & M & 53 & 10 \\
\hline H005C56 & M & 56 & 10 \\
\hline H006C41 & M & 41 & 10 \\
\hline M007C54 & F & 54 & 10 \\
\hline M008C55 & F & 55 & 10 \\
\hline H009C65 & $M$ & 65 & 10 \\
\hline H010C62 & $M$ & 62 & 10 \\
\hline H011C48 & $M$ & 48 & 10 \\
\hline H012C54 & $M$ & 54 & 3 \\
\hline H013C43 & $M$ & 42 & 3 \\
\hline M014C50 & $F$ & 50 & 3 \\
\hline H015C50 & $M$ & 50 & 3 \\
\hline H016C55 & $M$ & 55 & 3 \\
\hline H017C70 & $M$ & 70 & 3 \\
\hline H018C40 & $M$ & 40 & 3 \\
\hline M019C62 & F & 62 & 3 \\
\hline Promedio edad & & 52 & \\
\hline
\end{tabular}

Tabla 2. Individuos control

\begin{tabular}{|l|c|c|c|}
\hline $\begin{array}{c}\text { Código de } \\
\text { persona control }\end{array}$ & Sexo & $\begin{array}{c}\text { Edad } \\
\text { (años) }\end{array}$ & $\begin{array}{c}\text { Tiempo } \\
\text { Registro (min) }\end{array}$ \\
\hline M001-43 & F & 43 & 10 \\
\hline H002-21 & M & 21 & 10 \\
\hline H003-21 & M & 21 & 10 \\
\hline H004-22 & M & 22 & 10 \\
\hline H005-24 & M & 24 & 10 \\
\hline H006-23 & M & 23 & 10 \\
\hline H007-57 & M & 57 & 10 \\
\hline H008-22 & $M$ & 22 & 10 \\
\hline H009-24 & $M$ & 24 & 10 \\
\hline H010-53 & $M$ & 53 & 10 \\
\hline H011-42 & $M$ & 42 & 10 \\
\hline H012-43 & $M$ & 43 & 10 \\
\hline H013-51 & $M$ & 51 & 10 \\
\hline H014-44 & $M$ & 44 & 10 \\
\hline H015-15 & $M$ & 15 & 10 \\
\hline H016.42 & $M$ & 42 & 10 \\
\hline M017-22 & F & 22 & 10 \\
\hline M018-23 & F & 23 & 10 \\
\hline M019-21 & F & 21 & 10 \\
\hline Promedio Edad & & 32 & \\
\hline
\end{tabular}


Tabla 3. Parámetros electrocardiográficos de pacientes seropositivos a Chagas.

\begin{tabular}{|c|c|c|c|c|c|c|}
\hline $\begin{array}{c}\text { Código de } \\
\text { paciente }\end{array}$ & HR (LPM) & RR (s) & PR (s) & QRS (s) & QT (s) & QTc (s) \\
\hline H001C22 & 87,93 & 0,684 & 0,143 & 0,073 & 0,32 & 0,387 \\
\hline M002C49 & 78,73 & 0,765 & 0,131 & 0,067 & 0,336 & 0,384 \\
\hline M003C58 & 63,89 & 0,939 & 0,142 & 0,058 & 0,393 & 0,405 \\
\hline H004C53 & 84,05 & 0,714 & 0,188 & 0,087 & 0,333 & 0,394 \\
\hline H005C56 & 75 & 0,802 & 0,32 & 0,073 & 0,366 & 0,409 \\
\hline H006C41 & 84,08 & 0,717 & 0,13 & 0,068 & 0,316 & 0,373 \\
\hline M007C54 & 68,21 & 0,88 & 0,123 & 0,071 & 0,333 & 0,354 \\
\hline M008C55 & 61,52 & 0,977 & 0,168 & 0,077 & 0,394 & 0,399 \\
\hline H009C65 & 74,46 & 0,808 & 0,158 & 0,067 & 0,364 & 0.405 \\
\hline H010C62 & 67.26 & 0,894 & 0,18 & 0,108 & 0,365 & 0,387 \\
\hline H011C48 & 67,06 & 0,931 & 0,187 & 0,046 & 0,298 & 0,312 \\
\hline H012C54 & 81,53 & 0,737 & 0,144 & 0,049 & 0,342 & 0,400 \\
\hline H013C43 & 81,02 & 0,741 & 0,13 & 0,07 & 0,371 & 0,431 \\
\hline M014C50 & 71,3 & 0,842 & 0,12 & 0,081 & 0,367 & 0,400 \\
\hline $\mathrm{H} 015 \mathrm{C} 50$ & 69,48 & 0,869 & 0,143 & 0,06 & 0,282 & 0,302 \\
\hline H016C55 & 67,3 & 0,909 & 0,145 & 0,057 & 0,341 & 0,357 \\
\hline $\mathrm{H} 017 \mathrm{C} 70$ & 80,8 & 0,743 & 0,187 & 0,078 & 0,356 & 0,416 \\
\hline $\mathrm{H} 018 \mathrm{C} 40$ & 80,41 & 0,747 & 0,184 & 0,065 & 0,308 & 0,356 \\
\hline M019C62 & 58,03 & 1,035 & 0,165 & 0,089 & 0,406 & 0,400 \\
\hline Promedio & $73,79 \pm 8,58$ & $0,83 \pm 0,17$ & $0,16 \pm 0,04$ & $0,07 \pm 0,01$ & $0,346 \pm 0,033$ & $0,382 \pm 0,033$ \\
\hline
\end{tabular}

se aprecia que la Desviación Estandar (SD, Standar deviation) de la población control es mayor $(11,95)$ que la seropositiva $(8,58)$, lo cual infiere una mayor variabilidad de este parámetro en la población control.

El intervalo RR fue más corto en la población control, aunque no presentó diferencias significativas con la población seropositiva. Este intervalo determina la duración de un ciclo cardiaco. El intervalo PR, también conocido como PQ, contrario al parámetro anterior, fue más corto en la población seropositiva, sin mostrar diferencias significativas. Con respecto al complejo QRS, no se encontraron diferencias notables entre los grupos, ni significativas. Este tiempo fue mayor en la población seropositiva, pero al igual que en los anteriores parámetros, no se encontraron diferencias significativas. Por el contrario, el intervalo QTC, que lo hace con respecto a la frecuencia cardiaca, fue significativamente mayor $(P \leq 0,05)$ en los pacientes seropositivos.

\section{Parámetros de variabilidad de la frecuencia cardiaca}

Con respecto a los parámetros de dominio temporal, las tablas 5 y 6 muestran los resultados para pacientes seropositivos y controles respectivamente. La variable SDRR en la población control arrojó un promedio de $56,23 \pm 29,60 \mathrm{~ms}$, mientras que para los seropositivos a Chagas fue de 40,62 \pm 30,10 ms, observándose claramente unas desviaciones mayores en los primeros, aunque tanto la prueba de ANOVA como la T no establecieron diferencias significativas entre los grupos.

\section{Discusión}

La enfermedad de Chagas, es una enfermedad infecciosa que afecta significativamente la salud de la población humana a nivel mundial, especialmente en el continente suramericano, de donde es endémica. Entre los principales signos clínicos asociados a la croni- 
Tabla 4. Parámetros electrocardiográficos de personas control

\begin{tabular}{|c|c|c|c|c|c|c|}
\hline $\begin{array}{l}\text { Código de } \\
\text { personas control }\end{array}$ & HR (LPM) & RR (s) & PR (s) & QRS (s) & QT (s) & QTc (s) \\
\hline M001-43 & 62,72 & 0,956 & 0,156 & 0,062 & 0,36 & 0,368 \\
\hline $\mathrm{H} 002-21$ & 69,26 & 0,871 & 0,184 & 0,064 & 0,311 & 0,334 \\
\hline H003-21 & 74,79 & 0,808 & 0,146 & 0,069 & 0,32 & 0,356 \\
\hline H004-22 & 71,72 & 0,841 & 0,163 & 0,093 & 0,344 & 0,375 \\
\hline H005-24 & 83,29 & 0,723 & 0,16 & 0,07 & 0,325 & 0,383 \\
\hline H006-23 & 52,93 & 1,162 & 0,172 & 0,075 & 0,384 & 0,358 \\
\hline H007-57 & 69,99 & 0,861 & 0,178 & 0,049 & 0,341 & 0,368 \\
\hline H008-22 & 68,08 & 0,888 & 0,196 & 0,085 & 0,36 & 0,383 \\
\hline H009-24 & 85,37 & 0,704 & 0,182 & 0,064 & 0,322 & 0,384 \\
\hline H010-53 & 64,6 & 0,93 & 0,146 & 0,057 & 0,359 & 0,372 \\
\hline M011-42 & 72,49 & 0,829 & 0,165 & 0,07 & 0,337 & 0,371 \\
\hline H012-43 & 63,15 & 0,957 & 0,176 & 0,065 & 0,368 & 0,376 \\
\hline H013-51 & 62,16 & 0,968 & 0,154 & 0,044 & 0,351 & 0,357 \\
\hline H014-44 & 74,22 & 0,811 & 0,143 & 0,065 & 0,324 & 0,36 \\
\hline H015-15 & 85,88 & 0,702 & 0,129 & 0,064 & 0,317 & 0,379 \\
\hline H016.42 & 90 & 0,069 & 0,154 & 0,078 & 0,294 & 0,36 \\
\hline M017-22 & 103,5 & 0,581 & 0,105 & 0,082 & 0,289 & 0,38 \\
\hline M018-23 & 72,59 & 0,833 & 0,211 & 0,073 & 0,352 & 0,386 \\
\hline M019-21 & 64 & 0,94 & 0,215 & 0,068 & 0,354 & 0,365 \\
\hline Promedio & $73 \pm 11,95$ & $0,81 \pm 0,22$ & $0,17 \pm 0,03$ & $0,068 \pm 0,01$ & $0,337 \pm 0,025$ & $0,369 \pm 0,013$ \\
\hline
\end{tabular}

cidad de esta enfermedad, se encuentra la cardiopatía Chagásica, la cual es responsable de aproximadamente a 50.000 muertes cada año en su área de influencia en America, con variaciones en su mono de presentación, debido al genotipo infectivo del parásito, disponibilidad al tratamiento químico, vía de infección, entre otras.

En Colombia, los estudios con relación al análisis de los características electrocardiográficas de pacientes positivos a la enfermedad de Chagas, son pocos encontrándose, destacandose bloqueo completo de rama derecha $(40 \%)$, bloqueo aurículo-ventricular de segundo y tercer grado $(29,2 \%)$, disfunción del nodo sinusal $(28,3 \%)$, taquicardia ventricular $(23 \%)$, fibrilación auricular (19\%), hemibloqueo anterior izquierdo $(17,2 \%)$, flutter auricular $(3,3 \%)$ y bloqueo de rama izquierda $(3,3 \%)$. Estos hallazgos son similares a los reportados por Lázzari (1994); Luquetti (1997) y Rosas et al., (2002). En el presente estudio,la HR no arrojó diferencias significativas entre los grupos, pero se evidenció una mayor SD en los controles, lo cual indica una mayor variabilidad de este parámetro en este grupo. Los valores de HR son un poco más bajos que a los reportados por Octavio et al., (2004), quienes obtuvieron para pacientes chagásicos una HR de 79,5 $\pm 4,5$ Ipm, frente a los controles quienes registraron 75,0 \pm $5,2 \mathrm{lpm}$, registrando diferencias significativas entre los dos grupos. Hay que tener en cuenta que en el trabajo citado, la información se obtuvo durante 24 h a través de registro Holter. La menor HR en pacientes chagásicos ha sido explicada como consecuencia a una menor respuesta del nodo sinusal (Caeiro y Losa, 1994), a la estimulación autonómica o a la disautonomía que acompaña la enfermedad (Caeiro y Losa, 1994). El hecho que en este estudio no se encontraran diferencias significativas de este parámetro entre los grupos, probablemente se deba al tiempo de duración de los registros. 
Tabla 5. Parámetros de HRV en pacientes seropositivos a enfermedad de Chagas

\begin{tabular}{|c|c|c|c|}
\hline $\begin{array}{c}\text { Código de } \\
\text { paciente }\end{array}$ & HR (LPM) & $\begin{array}{c}\text { SDRR } \\
(\mathbf{m s})\end{array}$ & SD HR \\
\hline H001C22 & 87,94 & 34,6 & 4,52 \\
\hline M002C49 & 78,53 & 39,1 & 4,79 \\
\hline M003C58 & 63,89 & 21,7 & 1,47 \\
\hline H004C53 & 84,06 & 21,4 & 2,58 \\
\hline H005C56 & 75,01 & 35,1 & 3,32 \\
\hline H006C41 & 84,04 & 47,4 & 5,68 \\
\hline M007C54 & 68,11 & 26,5 & 2,08 \\
\hline M008C55 & 61,52 & 25,2 & 1,62 \\
\hline H009C65 & 74,46 & 39,9 & 4,85 \\
\hline H010C62 & 67,25 & 35,6 & 3,96 \\
\hline H011C48 & 67,11 & 148,9 & 16,85 \\
\hline H012C54 & 81,53 & 23,6 & 2,59 \\
\hline H013C43 & 81,02 & 25,7 & 2,85 \\
\hline M014C50 & 83,34 & 28,3 & 3,32 \\
\hline H015C50 & 69,34 & 64 & 5,75 \\
\hline H016C55 & 67,3 & 75,7 & 16,62 \\
\hline H017C70 & 80,8 & 17,6 & 1,89 \\
\hline H018C40 & 80,41 & 18,4 & 2 \\
\hline M019C62 & 57,61 & 38,4 & 2,08 \\
\hline
\end{tabular}

El intervalo RR, que determina la duración de un ciclo cardiaco, fue menor en los controles que en los chagásicos, aunque no se encontraron diferencias significativas sin embargo, en forma similar a lo observado con la HR, la SD fue mayor en los controles $(0,22)$ que en los pacientes $(0,17)$, lo cual implica una mayor dispersión de los datos y por ende, una variabilidad de la HR mayor. El hecho que no se hayan encontrado diferencias significativas en los valores de la HR, puede considerarse como explicación para que los tiempos del intervalo RR, tampoco presentaran diferencias, ya que como se mencionó, este parámetro representa la duración del ciclo cardiaco.

El complejo QRS, no arrojó diferencias significativas entre los grupos, pero fue ligeramente más bajo en la población control $(0,068 \pm 0,01 \mathrm{~s})$ que en la de pacientes chagásicos $(0,07 \pm 0,01 \mathrm{~s})$ y en ambos grupos, la duración del mismo, se encontró dentro del rango considerado como normal $(0,06-0,08 \mathrm{~s})$.

En los registros electrocardiográficos tomados a los pacientes chagásicos, se observó en el $68 \%$ de los seropositivos, extrasístoles ventriculares. La detección de extrasístoles ventriculares o de taquicardia ventricu-
Tabla 6. Parámetros de HRV en pacientes control

\begin{tabular}{|c|c|c|c|}
\hline $\begin{array}{c}\text { Código de } \\
\text { pacientes } \\
\text { control }\end{array}$ & HR (LPM) & SDRR (ms) & SD HR \\
\hline M001-43 & 62,72 & 55,3 & 3,77 \\
\hline H002-21 & 69,25 & 63,4 & 5,21 \\
\hline H003-21 & 74,8 & 69,2 & 6,46 \\
\hline H004-22 & 71,72 & 61,3 & 4,96 \\
\hline H005-24 & 83,31 & 38,4 & 4,48 \\
\hline H006-23 & 52,47 & 159,5 & 10,18 \\
\hline H007-57 & 69,99 & 53,4 & 4,37 \\
\hline H008-22 & 68,06 & 75,1 & 5,85 \\
\hline H009-24 & 85,39 & 31,2 & 3,66 \\
\hline H010-53 & 64,61 & 25,6 & 1,82 \\
\hline H011-42 & 72,5 & 37,1 & 3,23 \\
\hline H012-43 & 63,17 & 77,6 & 5,3 \\
\hline H013-51 & 62,15 & 44 & 4,61 \\
\hline H014-44 & 74,24 & 44,6 & 4,14 \\
\hline H015-15 & 85,93 & 46,9 & 5,51 \\
\hline H016-42 & 89,97 & 35,3 & 4,76 \\
\hline M017-22 & 103,54 & 29,5 & 5,04 \\
\hline M018-23 & 72,53 & 70 & 6,28 \\
\hline M019-21 & 64,01 & 51 & 3,65 \\
\hline
\end{tabular}

lar, se asocia con reducción en la expectativa de vida en los pacientes con enfermedad de Chagas (Santana 1987; Prata et al., 1974).

El intervalo QT no arrojó diferencias significativas entre grupos, pero se observó que el promedio fue mayor en los pacientes chagásicos, lo cual infiere un mayor tiempo en la conducción del impulso eléctrico a través del haz de His. Sin embargo al analizar el QTc, el promedio fue significativamente mayor $(\mathrm{P} \leq 0,05)$ en los pacientes seropositivos, lo cual confirma la existencia de un tiempo mayor en el recorrido del impulso eléctrico en este sector del sistema de conducción cardiaco. La dispersión del intervalo QT es considerado como un predictor de muerte súbita (Duque et al., 2007).

Aunque el objetivo de este trabajo no incluía la identificación de alteraciones electrocardiográficas relevantes en pacientes con enfermedad de Chagas, dado que este hecho está fuera de la competencia de los autores, es importante mencionar que existen predictores de riesgo de muerte súbita en estos pacientes, entre los que la literatura menciona: Disminución de la fracción de eyección del ventrículo izquierdo, evidencia de cardiomegalia en la radiografía de tórax (Rassi 
et al., 2007), dispersión del intervalo QT, inducibilidad de taquicardia ventricular en estudio electrofisiológico (Viotti R, 2005; Salles G, 2003), edad, diámetro sistólico del ventrículo izquierdo, trastornos de la conducción intraventricular y evidencia de taquicardia ventricular sostenida (Rosas et al., 2007).

La enfermedad en su evolución presenta arritmias severas que pueden desencadenar incluso la muerte súbita (González, 2001; Fuenmayor y Fuenmayor, 1996). La mortalidad a causa de la enfermedad de Chagas se relaciona con la presencia de compromiso cardíaco. La disfunción ventricular y falla cardíaca, arritmias ventriculares sostenidas, bradiarritmias y paro cardíaco previo predisponen a riesgo de muerte súbita, (Prata, 1974). La aparición de taquicardia ventricular no sostenida durante el ejercicio también es un factor de alto riesgo para muerte súbita en enfermedad de Chagas (Paola et al., 1995). Se sugiere que la fibrilación ventricular constituye el evento terminal en la mayoría de los casos de muerte súbita en la enfermedad de Chagas. Menos frecuente es la muerte por bradiarritmias o disociación electromecánica (Duque et al., 2007). Las alteraciones en la función simpática y parasimpática que se observan durante la evolución de la cardiomiopatía chagásica, están determinadas por un aumento precoz del tono simpático asociado a denervación cardiovagal progresiva relacionada con alteraciones inmunológicas específicas (Rassi et al., 2007; Salles et al., 2003; Leite et al., 2001).

Se puede concluir que tanto las variables estudiadas a partir de los registros ECG, como las correspondientes a la HRV, de los pacientes de este estudio indican que la enfermedad de Chagas de años de evolución, genera alteración de la función cardiaca que se traduce en un mayor incremento del QTc y de afectación de las variables del dominio de tiempo de la HRV (SDHR y SDRR), que resultan en valores más bajos para los seropositivos y que de acuerdo con la literatura consultada, estos menores valores de la HRV, están relacionados con alteraciones del manejo autónomo de la función cardiaca que se controla a través de las vías simpáticas y parasimpáticas, generando en los pacientes con enfermedad de Chagas, una mayor alteración parasimpática.

\section{Agradecimientos}

Los autores agradecen a la Universidad de los Llanos por el apoyo para el desarrollo de esta investigación suministrado a través de la Dirección General de Investigaciones de la misma universidad, al Dr. Omar Ramírez Gómez coordinador de Vigilancia en Salud del Departamento del Meta, también a todos y cada uno de los pacientes seropositivos a Chagas que de una forma voluntaria y desinteresada colaboraron con la presente investigación.

\section{Referencias}

Andrade ZA, Camara EJ, Sadigursky M, et al. Envolvimento do nódulo sinusal na doença de Chagas. Arq Bras Cardiol. 1998;50:153158

Bar-Yam Y. 1997. Dynamics of complex systems (Vol. 213). Reading, MA: Addison-Wesley.

Bestetti RB, Dalbo CM, Freitas QC, et al. Noninvasive predictors of mortality for patients with Chagas' heart disease: a multivariate stepwise logistic regression study. Cardiology. 1994;84:261

Boguccki S, Nosczczyk-Nowak A. Short.term heart rate variability (HRV) in healthy dogs. Pol J Vet Sci. 2015;18(2),307-312

Buzzano O, Mercado M, Lightowler C, perez E, Maldana H. Predicción de fenómenos arrítmicos a través de la variabilidad de la frecuencia cardiaca en caninos. Cardiologia canina, Revista Veterinaria. 2012;23(1):55-58

Caeiro T, losa D. Chronic Chagas' disease: possible mechanism of sinus bradycardia. Can J Cardiol. 1994;10:765-768

Cuenca G. 2007. Ecocardiograma en Enfermedad de Chagas. En: Rosas F, Vanegas D y Cabrales M. (Editores). Enfermedad de Chagas, $1^{\underline{a}}$ Ed, Sociedad Colombiana de Cardiología, Bogotá, Colombia, p. 71-76

Duque M, Medina L, Uribe W, Marín J, Gil E. 2007. Muerte súbita en enfermedad de Chagas. En: Rosas F, Vanegas D y Cabrales M. (Editores). Enfermedad de Chagas, $1^{\underline{a}}$ Ed, Sociedad Colombiana de Cardiología, Bogotá, Colombia, p. 95-102

Fuenmayor AJ, Fuenmayor AM. Sudden death in patient with chagasic myocarditis. Arch Ins Cardiol Mex. 1996;2:157-161

González-Zuelgaray J. Arritmias ventriculares en la enfermedad de Chagas. Rev Col Arg Cardiol. 2001;5:14

Guillén P, Jugo D, Carrasco H, Rojas R. Dinámica no lineal para caracterizar la variabilidad del ritmo cardíaco en pacientes chagásicos agudos, Ciencia. 2003;11(4):276-283

Guhl F. 1998. Estado actual del control de la enfermedad de Chagas en Colombia. Editores. Curso-taller control de tripanosomiasis americana y leishmaniasis: aspectos biológicos, genéticos y moleculares. Corcas Editores, Bogotá: 47-81

INS-Instituto Nacional de Salud/OPS- Organización Panamericana de la Salud, 2009. Guía Protocolo para la vigilancia en salud pública de Chagas. p.48 [Consultada julio de 2017]. http://www. ins.gov.co/temasdeinteres/Chagas/01\%20Protocolo\%20Chagas.pdf

Jugo D, Medina R, Rojas R, Schlegel T, Arenare B. Aplicación de nuevos métodos de análisis de la variabilidad de la Frecuencia Cardiaca en pacientes chegásicos crónicos, Universidad, Ciencia y Tecnología. 2008;12(48):129-136 
Khor K, Shiels I, Campbell F, Greeer R, Rose A, Mills P. Evaluation of a technique to measure heart rate variability in anaesthetized cats. Vet J. 2014;199(1):229-235

Laranja FS, Dias E, Nobrega G, Miranda A. Chagas' disease; a clinical, epidemiologic and pathologic study. Circulation. 1956;14(6):1035-1060

Lázzari JO. Autonomic nervous system alterations in Chagas disease. Review of the literature. In: PAHO-Panamerican Health Organization; Aci Pub Eds. Chagas and the Nervous System p. 1994;547:72-96

Leite LR, Fenelon G, Paes AT, de Paola AA. The impact of syncope during clinical presentation of sustained ventricular tachycardia on total and cardiac mortality in patients with chronic chagasic heart disease. Arq Bras Cardiol; 2001;77:439-452

Luquetti OA. 1997. Tratamento etiológico da doença de Chagas. Ministério da Saude, Brasil, Brasilia.

Lightowler C. 2011. Electrocardiografia ambulatoria (Holter). En: Electrocardiografia Veterinaria, 2da Ed, Buenos Aires, pp 171183

Mora M, Barrio A, Nasser J, Sánchez O, Marco D, Gonorazki J, et al. Contribution of the molecular biology technics at Chagas' disease. Bol Acad Nac Med B Aires. 1998;76(1):103-118

Paola A, Gomez J, Terzian A, et al. Ventricular tachycardia during exercise testing as a predictor of sudden in patients with chronic chagasic cardiomyopathy and ventricular arrhythmias. $\mathrm{Br}$ Med J. 1995;74:293-295

PAHO-Pan American Health Organization / WHO- World Health Organization. 2017. General Information-Chagas Disease. [Consultada Julio de 2017] http://WWW.paho.org/hg/index. php?option=com_topics\&view=article\&id=10\&ltemid

Prata A, Andrade Z, Guimaraes A. 1974. Chagas' heart disease. In: Shaper AG, Hutt M, Fejfar Z. Cardiovascular disease in the tropics. London: British Medical Association. p. 264-81

Rasmussern C, Falk T, Domanjko A, Schaldemose M, Zois N, Moesgaard $S$, et al. Holter monitoring of small breed dogs with advanced myxomatous mitral valve disease with and without a history of syncope. J Vet Intern Med. 2014;28(1):363-370

Rassi A Jr, Rassi A, Rassi SG. Predictors of mortality in chronic Chagas disease: a systematic review of observational studies. Circulation. 2007;115:1101-1108

Restrepo C. 2006. Variabilidad de la frecuencia cardiaca: Cambio de vocación para una prueba. En: Cabrales M, Vanegas D. Manual de métodos diagnósticos en electrofisiología cardiovascular. Bogotá, pp 93-97

Richman JS, Moorman JR. Physiological time-series analysis using approximate entropy and sample entropy. Am J Physiol Heart Circ Physiol. 2000;278(6):H2039-H2049

Rodas G, Carballido C, Ramos J, Capdevila L. Variabilidad de la frecuencia cardiaca: concepto, medidas y relación con aspectos clínicos (i), Archivos de medicina del deporte (AMD). 2008;15(123):41-47

Rosas F, Guhl F, Velasco V, et al., 2007. Cardiomiopatia Chagásica. En: En: Rosas F, Vanegas D y Cabrales M. (Editores). Enfermedad de Chagas, 1a Ed, Sociedad Colombiana de Cardiología, Bogotá, Colombia, p. 47-62

Rosas F, Velasco V, Jumbo L, Rodríguez D, Arboleda F, JaramiIlo $\mathrm{C}$, et al. Cardiomiopatía de Chagas. Acta Med Colomb. 2002;27:26-40

Rosas F, Melgarejo I, Jumbo L, Velasco V, Jaramillo C, Rodríguez D, et al. 2000. Echocardiographic features of Chagas disease. Europace; 1:D89

Rosas J, Velasco V, López J, Guhl F, Correa J, Treut G, Arboleda $\mathrm{F}$, et al. Variable forma de presentación de la cardiomiopatía chagásica en Colombia. Rev Patol Trop Goias. 1998;27:35-36

Salles G, Xavier S, Sousa A, et al. Prognostic value of QT interval parameters for mortality risk stratification in Chagas' disease: results of a long-term follow-up study. Circulation. 2003;108:305312

Sánchez J, Parrado E, Capdevila L. Variabilidad de la frecuencia cardiaca y perfiles psicológicos en deportes de equipo de alto rendimiento. Rev Psicol Deporte. 2013;22(2):345-352

Santana O. 1987. Arritmia ventricular e evoluzao clínica de pacientes na fase crónica da doenza de Chagas. Tese de Mestrado. Facultade de Medicina da Universidade Federal da Bahia. Salvador; 50

Sterin-Borda L, Borda E. Role of neurotransmitter autoantibodies in the pathogenesis of chagasic peripheral dysautonomia. Ann NY Acad Sci. 2000;917: 273-280

Tundo F, Lombardi F, Rocha MC, Botoni F, Schmidt G, Barros VC, et al. Heart rate turbulence and left ventricular ejection fraction in Chagas disease. Europace. 2005;7:197-203

Viotti R, Vigliano C, Lococ B, Petti M, et al. Indicadores clínicos de la progresión de la miocarditis chagásica crónica. Rev Esp Cardiol. 2005;58:1037-1044

José Corredor:https://orcid.org/0000-002-2434-7928 Fernando Riveros:https://orcid.org/0000-0001-8948-6956 